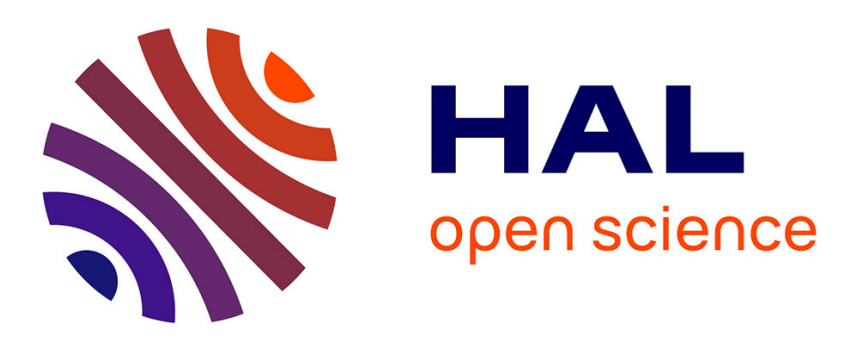

\title{
Dispositif utilisant la transmission hyperfréquence pour mesurer la durée de vie des porteurs injectés dans un échantillon semi-conducteur à basse température. Application à la diode pin
}

\author{
M. Brousseau, J. Barrau, J.C. Brabant
}

\section{To cite this version:}

M. Brousseau, J. Barrau, J.C. Brabant. Dispositif utilisant la transmission hyperfréquence pour mesurer la durée de vie des porteurs injectés dans un échantillon semi-conducteur à basse température. Application à la diode pin. Revue de Physique Appliquée, 1971, 6 (1), pp.1-4. 10.1051/rphysap:01971006010100 . jpa-00243498

\section{HAL Id: jpa-00243498 \\ https://hal.science/jpa-00243498}

Submitted on 1 Jan 1971

HAL is a multi-disciplinary open access archive for the deposit and dissemination of scientific research documents, whether they are published or not. The documents may come from teaching and research institutions in France or abroad, or from public or private research centers.
L'archive ouverte pluridisciplinaire HAL, est destinée au dépôt et à la diffusion de documents scientifiques de niveau recherche, publiés ou non, émanant des établissements d'enseignement et de recherche français ou étrangers, des laboratoires publics ou privés. 


\title{
REVUE DE PHYSIQUE APPLIQUÉE
}

\author{
Supplément au « Journal de Physique *
}

\section{DISPOSITIF UTILISANT LA TRANSMISSION HYPERFRÉQUENCE POUR MESURER LA DURÉE DE VIE DES PORTEURS INJECTÉS DANS UN ÉCHANTILLON SEMI-CONDUCTEUR A BASSE TEMPÉRATURE APPLICATION A LA DIODE PIN}

\author{
M. BROUSSEAU, J. BARRAU et J. C. BRABANT
}

Laboratoire de Physique des Solides, associé au C. N. R. S.

Faculté des Sciences et I. N. S. A.,

118, route de Narbonne, 31, Toulouse (04)

(Reçu le 16 juillet 1970)

\begin{abstract}
Résumé. - Nous présentons un ensemble expérimental destiné à l'étude de processus de recombinaison des porteurs injectés dans un matériau semiconducteur : il utilise l'absorption d'une onde électromagnétique hyperfréquence par les porteurs libres. Ce dispositif, actuellement appliqué à l'étude de la recombinaison des porteurs injectés dans la zone intermédiaire de structures PIN permet des mesures de durées de vie égales ou supérieures à $4 \times 10^{-9} \mathrm{~s}$ la température étant supérieure à $4^{\circ} \mathrm{K}$.
\end{abstract}

Abstract. - We describe a set up used for studies of recombination processes injected carriers in semiconductors : the principle of the method is the transient absorption of microwaves by free carriers. This apparatus, presently applied for measurements in PIN structures, allows the determination of carrier lifetimes above $4 \times 10^{-9} \mathrm{~s}$ when the temperature is chosen above $4.2^{\circ} \mathrm{K}$.

I. Principe de la méthode. - Le principe a été décrit précédemment [1]. Rappelons brièvement que si l'on place dans un guide d'onde un échantillon semiconducteur la transmission hyperfréquence peut être calculée en utilisant la matrice d'un quadripôle linéaire. Cette matrice est fonction de la géométrie $(G)$, de la constante diélectrique complexe $\tilde{\varepsilon}$ de l'échantillon dans le guide, de la pulsation $\omega$ de l'onde hyperfréquence. Par suite, le signal $V_{\mathbf{H}}$ sur le détecteur hyperfréquence terminal dépend de la puissance incidente $P_{\mathrm{i}}$ ainsi que de $G, \widetilde{\varepsilon}, \omega$. Travaillant à $P_{\mathrm{i}}, G, \omega$ fixés, $V_{\mathrm{H}}$ dépend de $\widetilde{\varepsilon}=\varepsilon_{\mathrm{D}}-n f(\tau)$ où $\varepsilon_{\mathrm{D}}$ est la contribution du réseau à la constante diélectrique tandis que $f(\tau)$, donnée par les propriétés de transport du semiconducteur [2], est supposée constante pendant la phase transitoire de recombinaison qui suit une injection stationnaire (les porteurs sont à la température du réseau dès que l'on a supprimé l'excitation); $n$ est la densité de porteurs.

Il découle pendant la phase transitoire :

$$
-\frac{n}{\frac{\mathrm{d} n}{\mathrm{~d} t}}=-K\left(V_{\mathrm{H}}\right) \frac{1}{\frac{\mathrm{d} V_{\mathrm{H}}}{\mathrm{d} t}}
$$

avec

$$
K\left(V_{\mathrm{H}}\right)=n \frac{\mathrm{d} V_{\mathrm{H}}}{\mathrm{d} n}
$$

$K\left(V_{\mathbf{H}}\right)$ est la fonction de calibration qui permet de déduire la durée de vie de la mesure de la pente du signal transitoire $\mathrm{d} V_{\mathrm{H}} / \mathrm{d} t$ (Fig. 1); Les mesures relatives de durée de vie qui permettent d'obtenir par exemple la loi de variation avec la température ou bien avec la dose d'irradiation, etc... sont possibles en faisant la mesure de pente $\mathrm{d} V_{\mathrm{H}} / \mathrm{d} t$ lorsque $V_{\mathrm{H}}$ passe par une valeur fixée au cours de la phase transitoire de recombinaison.

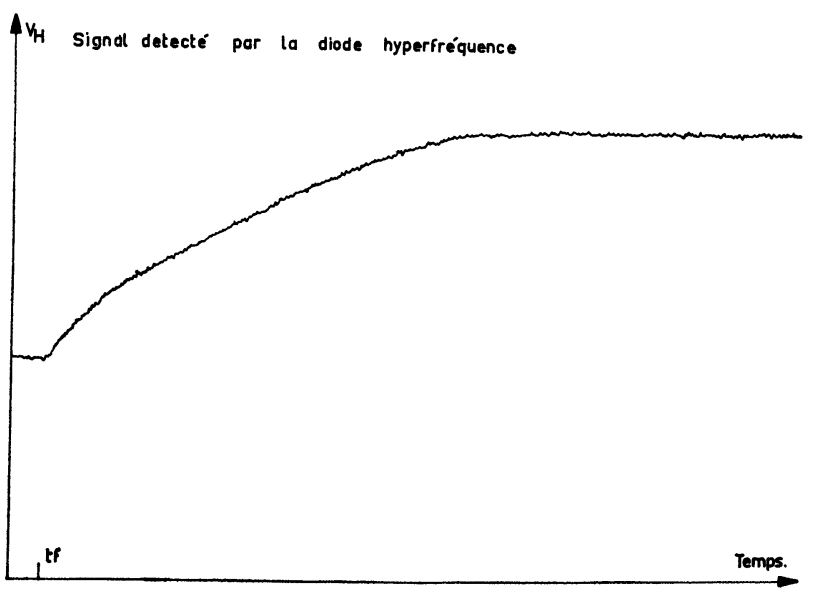

Fig. 1. - Enregistrement du signal hyperfréquence au cours d'une phase transitoire de recombinaison des porteurs dans la zone centrale d'une structure PIN; l'instant $t_{t}$ marque la fin de l'injection stationnaire des porteurs

$$
X=25 \mathrm{~ns} / \mathrm{cm} \quad Y=12,5 \mathrm{mV} / \mathrm{cm} \text {. }
$$


Les mesures absolues nécessitent la détermination expérimentale de la fonction de calibration $K\left(V_{\mathrm{H}}\right)$ : en se plaçant dans des conditions d'injection stationnaire et pour le cas fréquent $\omega \tau \ll 1$, le produit $\sigma_{0} \mathrm{~d} V_{\mathrm{H}} / \mathrm{d} \sigma_{0}$ représente $K\left(V_{\mathrm{H}}\right)$ et se déduit du relevé expérimental de la fonction $V_{\mathrm{H}}\left(\sigma_{0}\right)$. Ce dernier relevé est réalisé en choisissant une série de niveaux d'injection stationnaires distincts correspondant à diverses conductivités injectées $\sigma_{0}$.

Dans le cas de la mesure de la durée de vie des porteurs injectés dans la zone centrale d'une structure PIN, - l'onde hyperfréquence se propageant dans la zone $\mathrm{I}-$, la conductivité $\sigma_{0}$ injectée est déterminée par le choix du courant direct ; elle est mesurable par une méthode impulsionnelle précédemment décrite [3].

II. Dispositif expérimental. — L'ensemble expérimental comprend :

II. 1 Le CRYOSTAT A IRRAdiation (Fig. 2). - Conçu pour permettre des irradiations à $4,2 \circ \mathrm{K}$ par des

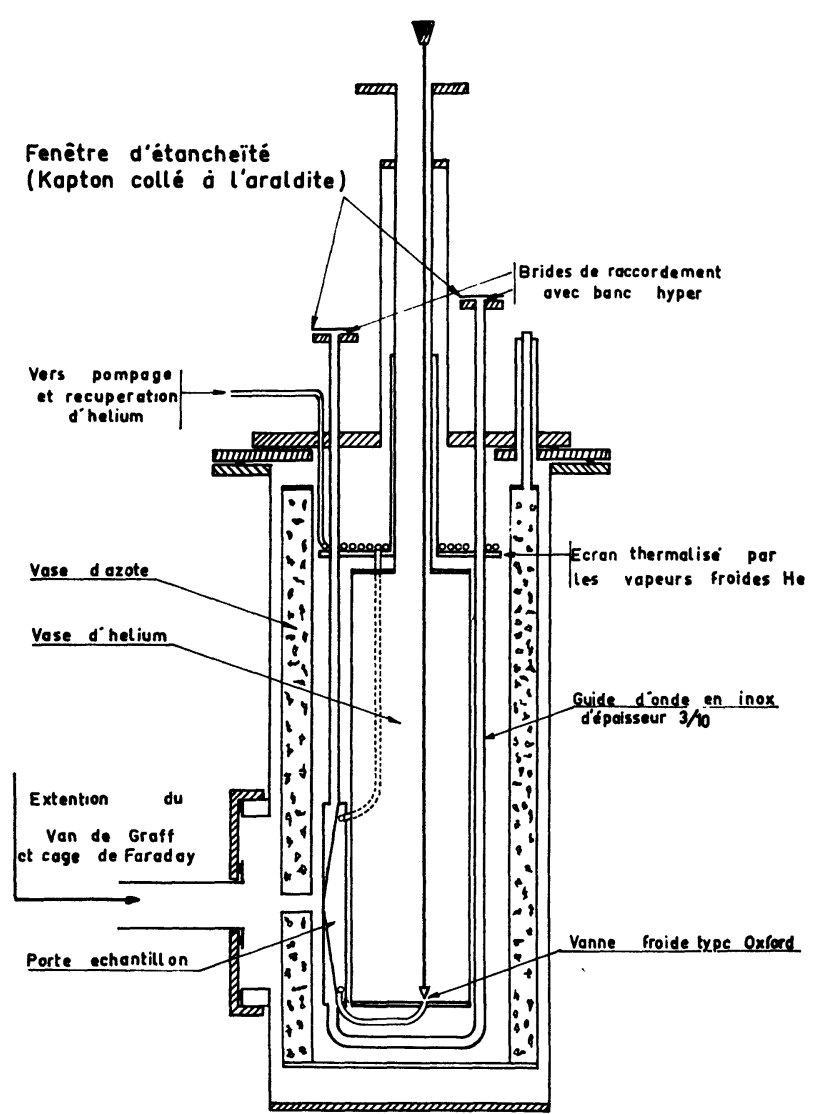

Fig. 2. - Schéma du cryostat à irradiation à température variable.

électrons de $2 \mathrm{MeV}$, ainsi que des recuits à diverses températures entre $4,2 \circ \mathrm{K}$ et l'ambiante, le cryostat est constitué d'un vase à hélium liquide d'une contenance de 2,5 litres entouré d'un vase annulaire rempli d'azote liquide (3 litres). En période de repos - hors recuit, hors irradiation - son autonomie est d'une quinzaine d'heures.
Située au fond du vase à hélium et commandée de l'extérieur, une vanne froide - type Oxford Inst. assure un écoulement à débit réglable de l'hélium liquide vers l'échangeur situé dans le porte-échantillon. Quand la vanne est grande ouverte, le porte-objet est plein d'hélium liquide et l'échantillon se thermalise à $4,2{ }^{\circ} \mathrm{K}$ pourvu que soient prises certaines précautions pour limiter l'apport de chaleur par le flux d'électrons irradiant et par le courant impulsionnel traversant l'échantillon au moment des mesures [3].

En outre par une fermeture appropriée de la vanne et une dépression variable réalisée en aval de la chambre d'échange, un courant d'hélium gazeux froid apporte à l'échantillon les frigories nécessaires à la régulation en température dont le principe est décrit plus loin.

Le gaz froid après passage dans le porte-objet sert à thermaliser l'écran supérieur solidaire du vase d'hélium.

Un ensemble de trois ouvertures, sur le porteéchantillon, dans le vase à azote et dans l'enceinte extérieure, permet d'irradier l'échantillon in situ. Aucune fenêtre n'obture ces ouvertures : le cryostat, entièrement démontable, possède un vide d'isolement commun avec le Van de Graaff utilisé pour les irradiations.

II. 2 LA RÉGUlATION DE TEMPÉRATURE (Fig. 3). Elle utilise deux thermocouples différentiels cuivre/orcobalt dont la jonction froide est soudée sur le fond du vase à hélium.

Le premier sert à enregistrer la courbe de température de l'échantillon.

L'écart entre la f. e. m. fournie par le second et une tension d'opposition délivrée par un pont de tension AOIP est amplifié par un amplispot Sefram (AGT) et introduit sur l'une des deux voies d'un enregistreur graphirac Sefram (BGD). On recueille donc sur le potentiomètre attaché au suiveur de spot une tension proportionnelle à l'écart entre la f. e. $\mathrm{m}$ du thermocouple et celle correspondant à la température que l'on veut atteindre. Cette tension est adressée à un régulateur Transireg-Schlumberger qui permet le choix d'une action proportionnelle, intégrale ou dérivée du signal reçu et agit sur un amplificateur de puissance fournissant le chauffage nécessaire sur un thermocoax Philips soudé au porte-objet.

Cet ensemble, utilisé en corrélation avec l'ensemble de pompage permet de réaliser tout palier de température entre $4^{\circ} \mathrm{K}$ et $300^{\circ} \mathrm{K}$. La vitesse de montée pour les 5 derniers degrés est au maximum de $10 \mathrm{~s}$ (vers $100{ }^{\circ} \mathrm{K}$ ) tandis que la vitesse de descente pour les 5 premiers degrés est d'environ $1 \mathrm{~s}$. La stabilisation est assurée, quelle que soit la température, à mieux que $1 / 10$ de degré près.

La consommation moyenne d'hélium pour une semaine de travail comportant des mesures de préirradiation, l'irradiation, les recuits jusqu'à la température ambiante est de 60 litres environ. 


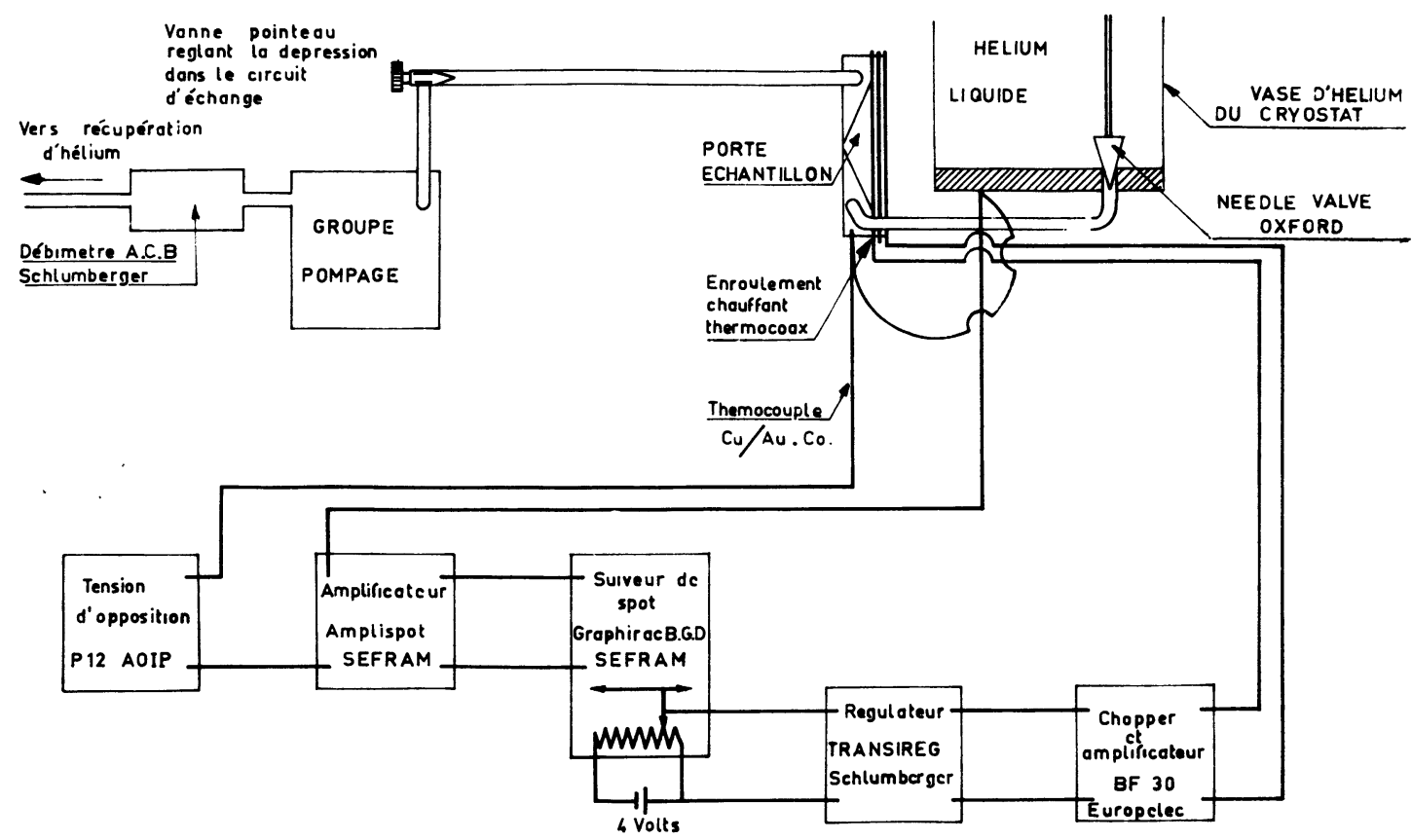

FIG. 3. - Ensemble de régulation de la température.

II. 3 L'APPAREILLAGE HYPERFRÉQUENCE (Fig. 4.) C'est un montage classique de banc hyperfréquence en bande $\mathrm{X}$ avec pont déphaseur améliorant la sensibilité de détection.

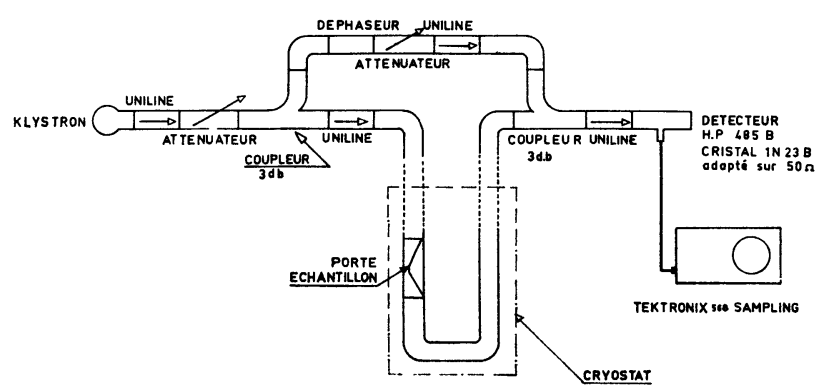

Fig. 4. - Schéma du dispositif hyperfréquence.

Le générateur est un klystron $2 \mathrm{~K} 25$ centré sur $9800 \mathrm{MH}$; le détecteur, un crystal 1 N 23 B adapté sur $50 \Omega$ et monté sur un support Hewlett Packard débarrassé de ses connexions selfiques et capacitives de manière à préserver les temps de montée et de descente des signaux détectés.

Notons la présence d'unilines avant et après l'échantillon qui favorisent l'adaptation de l'onde hyperfréquence.

La partie du banc située à l'intérieur du cryostat, en guide d'inox de 3/10 d'épaisseur (Métaux Inoxydables Ouvrés), est en communication avec le vide d'isolement du cryostat ; l'étanchéité est assurée par de la feuille de Kapton de $50 \mu$ collée à l'araldite sur 2 brides de raccordement bien polies.

II. 4 LE PORTE-OBJet (Fig. 5). - Le porte-objet présenté ici est destiné à l'étude de la durée de vie des porteurs injectés dans la zone centrale de structures $\mathrm{P}^{+} \mathrm{IN}^{+}$. a)

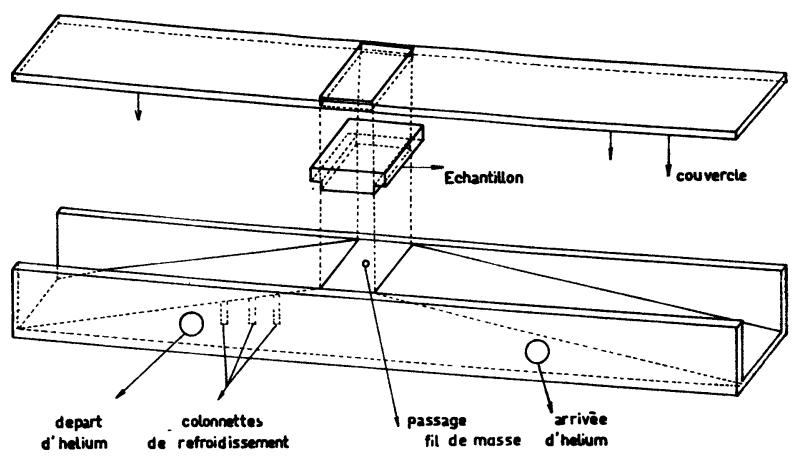

b)

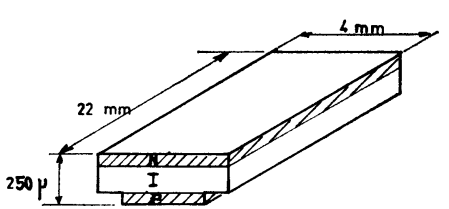

FIG. 5a. - Porte-objet et chambre d'échange thermique. b. - Diode PIN préparée pour être positionnée dans le porteobjet : les bords de la zone $\mathrm{P}^{+}$ont été enlevés par sablage de sorte que l'injection des porteurs se fait dans une zone uniformément irradiée.

Nous disposons de plaquettes d'épaisseur 250, 450 et $600 \mu$-zones dégénérées $\mathrm{N}^{+}$et $\mathrm{P}^{+}$comprises de $50 \mu$ chacune - découpées aux dimensions de $4 \times 22 \mathrm{~mm}$. L'onde hyperfréquence doit être guidée de façon à traverser la zone centrale mince dans sa petite dimension. Pour cela le porte-objet est constitué de 2 parties:

- Un bloc de cuivre en forme de "toit» assure l'adaptation au guide standard d'inox à ses extrémités.

- Un couvercle en cuivre comporte un dégagement 
destiné à recevoir l'échantillon au centre (chaque calibre d'échantillon nécessite un couvercle particulier).

Le parfait usinage de ce porte-objet est très important afin d'offrir d'une part la meilleure adaptation d'impédance hyperfréquence, d'autre part le meilleur positionnement de l'échantillon dans l'espace qui lui est réservé. Ce résultat est obtenu au moyen d'un usinage soigné par étincellage de l'ensemble des pièces.

Dans la masse même du bloc de cuivre porte-objet une chambre d'échange thermique constituée de chicanes a été réalisée; elle permet la thermalisation rapide de l'échantillon.

Notons enfin que du point de vue électrique le porteéchantillon constitue la masse de l'ensemble électronique : le côté $\mathrm{P}^{+}$des diodes y est raccordé tandis que le côté $\mathrm{N}^{+}$est isolé du couvercle au moyen d'une feuille mince de Kapton ( $5 \mu$ d'épaisseur).

II . 5 L'ENSEMBLE ÉLECTRONIQUE DESTINÉ A L'INJECTION DES PORTEURS ET AUX MESURES (Fig. 6). - L'objet

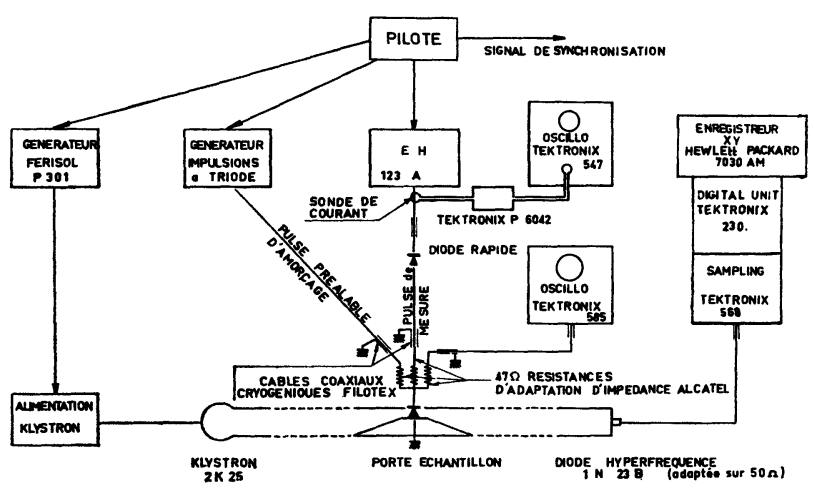

FIG. 6. - Schéma du dispositif électronique destiné à l'injection des porteurs et aux mesures.

principal du dispositif électronique est la création de signaux carrés à temps de montée et de descente faibles (voisin de $5 \mathrm{~ns}$ ), leur acheminement sans détérioration des générateurs d'impulsion vers l'échantillon, le prélèvement de signaux de mesure rapides et leur lecture sur des oscilloscopes en temps réel et à échantillonnage.

Nous disposons actuellement d'un générateur E. $\mathrm{H}$. à faible temps de montée ( $<7 \mathrm{~ns})$ délivrant 50 volts dans $50 \Omega$ et d'un générateur réalisé au laboratoire dont les caractéristiques principales sont une impédance de sortie très élevée, un temps de montée court et une puissance crête très élevée.

L'adaptation électrique de l'échantillon dont l'impédance est faible en régime de conduction ( 2 à $3 \mathrm{ohms}$ ) est assurée par des résistances (choisies au voisinage de $47 \Omega$ ) à couches évaporées d'alliages métalliques fournies par Alcatel et dont le coefficient de température entre 4 et $300^{\circ} \mathrm{K}$ est très faible.

Le transport des signaux dans le cryostat est assuré par des câbles cryogéniques Filotex de $50 \Omega$.

Dans le raccordement des câbles, il importe évidemment de soigner particulièrement le circuit de masses : Pour cela, nous avons rapporté toutes les masses en étoile vers le bloc porte-objet, ce qui impose de découpler à l'aide de capacités ou de transformateurs d'impulsions les liaisons entre appareils - en particulier les liaisons de synchronisation.

Le signal transitoire hyperfréquence de recombinaison est enregistré directement sur papier millimétrique (enregistreur Hewlett-Packard) ; un digital unit Tecktronix permet un affichage de la pente $\mathrm{d} V_{\mathrm{H}} / \mathrm{d} t$ au niveau $V_{\mathrm{H}}$ choisi.

III. Conclusion. - Grâce à cet ensemble, nous avons pu mesurer des durées de vie égales ou supérieures à $4 \times 10^{-9} \mathrm{~s}$ sur des diodes PIN au silicium entre $4,2{ }^{\circ} \mathrm{K}$ et l'ambiante, avant et après irradiation par des électrons de 1,5 MeV.

Nous avons pu observer et analyser des oscillations de recombinaison qui interviennent à fort niveau d'injection dans les structures $\mathrm{P}^{+} \mathrm{PN}^{+}$à basse température $(4,5)$.

L'appareillage est actuellement utilisé à l'étude des sections de capture des centres recombinants.

\section{Bibliographie}

[1] Brousseau (M.) et Schuttler (R.), Solid-St. Electron., $1969,12,417$.

[2] Smith (R. A.), Semi-conductors (édité par Cambridge Université Press), 1959.

[3] Brousseau (M.), Barrau (J.) et Brabant (J. C.), Appl. phys. Lett., 1970, 17, 297.
[4] Brousseau (M.), Barrau (J.), Brabant (J. C.), et NGuYen van TuYen, Solid-St. Electron., 1970, 13, 906.

[5] Brousseau (M.), Thèse, Contribution à l'étude de la double injection dans le silicium, Toulouse, mars 1970. 